\title{
MAPEO DE LOS BOSQUES TIPO VARILLAL UTILIZANDO IMÁGENES DE SATÉLITE RAPIDEYE EN LA PROVINCIA MAYNAS, LORETO, PERÚ
}

Juan PALACIOS ${ }^{1}$, Ricardo ZÁRATE ${ }^{1}$, Guiuseppe TORRES ${ }^{1}$, Jean-P. DENUX ${ }^{2}$, José MACO ${ }^{1}$, George GALLARDO $^{3,4}$, Tony MORI ${ }^{5}$, Jhon RENGIFO ${ }^{1}$, Anita JARAMA ${ }^{1}$, María MARÍN ${ }^{1}$, Franco GARCÍA ${ }^{1}$, Angela CUADROS

1 Instituto de Investigaciones de la Amazonía Peruana. Programa de Investigación en Cambio Climático Desarrollo Territorial y Ambiente (PROTERRA); Av. Quiñones km 2.5, San Juan Bautista, Maynas, Loreto, Perú. jpalacios@iiap.org.pe

2 Université de Toulouse, INPT, Ecole d'Ingénieurs de Purpan, UMR 1201 Dynafor, 75 Voie du TOEC, F-31076 Toulouse Cedex 03, Francia.

3 Centro para el Desarrollo del Indígena Amazónico (CEDIA). Calle Las Camelias 162, San Juan Bautista, Maynas, Loreto, Perú.

4 Gobierno Regional de Loreto. Programa Regional de Manejo de Recursos Forestales y Fauna Silvestre. Av. A. Quiñones km 1.5, Belén, Maynas, Loreto, Perú.

5 Organismo de Supervisión de los Recursos Forestales y de Fauna Silvestre (OSINFOR). Av. Javier Prado Oeste 694, Magdalena del Mar, Lima, Perú.

\section{RESUMEN}

Los bosques tipo varillal albergan una diversidad biológica endémica y varias especies monodominantes, cuando estos bosques se ubican en los pantanos acumulan gran cantidad de carbono en el suelo. El objetivo del presente estudio fue elaborar el mapa de distribución espacial de los varillales sobre arena blanca y sobre pantano en la provincia Maynas, Loreto, a escala 1/100,000, utilizando imágenes de satélite RapidEye y aplicando el algoritmo clasificador de Máxima Verosimilitud (MVS) en el programa ArcGIS versión 10.1. Usamos siete categorías de clasificación en tres escenas RapidEye y definimos las firmas espectrales respectivas basadas en 32 áreas de entrenamiento de muestreo en campo. Se clasificaron las 206 escenas restantes utilizando el algoritmo MVS. El mapa fue validado utilizando 57 puntos de control entre varillales y otros tipos de bosque. Se determinaron 51,025 ha de varillales que representan $0.67 \%$ de la superficie de la provincia Maynas, distribuidas principalmente entre las cuencas de los ríos Napo y Nanay y entre la ciudad de Iquitos y la boca del Río Amazonas. Obtuvimos un $91.2 \%$ de exactitud en la clasificación de los varillales. Las bandas borde rojo (BR) e ifrarrojo cercano (IRC) de las imágenes RapidEye diferenciaron mejor los varillales de los otros bosques. Se reportan nuevas áreas de bosque de varillales sobre arena blanca y pantanos. En conclusión, las imágenes RapidEye son útiles para clasificar y diferenciar los varillales de otros tipos de bosque, siendo el mapa de los varillales útil para la exploración de nuevos sitios en el estudio de almacenamiento de carbono en el suelo.

PALABRAS CLAVE: bosques sobre arena blanca, RapidEye, SIG, teledetección, varillal hidromórfico.

\section{MAPPING OF VARILLAL FOREST USING RAPIDEYE SATELLITE IMAGES IN THE PROVINCE OF MAYNAS, LORETO, PERU}

\begin{abstract}
The varillal forest type has an endemic biodiversity and several monodominant species, when occurring in swampy areas these forests have great amounts of carbon accumulated in the soil. The aim of this study was to develop a distribution map of varillales occurring on white sand and swamps in the province of Maynas, Loreto at scale of 1/100,000, using RapidEye satellite imagery and applying the classifier algorithm of Maximum Likelihood (ML) in the program ArcGIS version 10.1. We used seven classification classes in three RapidEye scenes, and defined the respective spectral signatures based on 32 training areas with reference plots sampled in the field. The remaining 206 scenes were classified using the ML algorithm. The map was validated using 57 check points of varillales and other forest types. We determined 51,025 ha of varillales representing $0.67 \%$ of the province of Maynas; these areas are distributed mainly between the basins of the Napo and Nanay rivers, and between Iquitos and the mouth of the Amazon River. The map of varillales was validated to $91.2 \%$. The red-edge and near infrared bands of the RapidEye images differentiated varillales from other forest types. New areas of varillales on white sand and swamps are reported. In conclusion, RapidEye images are useful to classify and differentiate varillales from other forest types, while this map of varillales is useful for the exploration of new study areas of below-ground carbon.
\end{abstract}

KEYWORDS: White-sand forest, RapidEye, GIS, Remote Sensing, pole forest. 


\section{INTRODUCCIÓN}

Los varillales comprenden comunidades vegetales que en el Perú solo se encuentran en el departamento de Loreto. Los varillales sobre arena blanca se encuentran principalmente en áreas de las reservas nacionales de Allpahuayo Mishana y Matsés, así como en Jeberos y en las cuencas de los ríos Nanay, Tapiche y Pastaza-Morona. Los varillales sobre pantanos, denominados varillales hidromórficos, se presentan principalmente en la región comprendida entre el oeste del Río Tigre y el norte del Río Marañón (Draper et al., 2014). Estas comunidades se caracterizan por su vegetación escleromórfica con especies de tallos delgados de baja a mediana estatura. Ocurren en sustratos de baja cantidad de nutrientes y $\mathrm{pH}$ ácido, dando origen a una composición florística poco diversa y monoespecífica (Fine et al., 2006). Mientras los varillales sobre arena blanca se desarrollan en sustratos cuarcíticos, los varillales hidromórficos se desarrollan sobre pantanos donde existe gran acumulación de materia orgánica en forma de turba y no cuentan con sustratos arenosos (Zárate et al., 2013; Draper et al., 2014).

Los varillales se caracterizan por presentar una composición florística muy peculiar, endémica, monodominante y poco diversa (Fine et al., 2006; Fine et al., 2010; Vicentini, 2004; Kelly et al., 2014). Por ejemplo, Jacqueshuberia loretensis es una especie endémica de los varillales sobre arena blanca, mientras Caraipa utilis, y Pachira brevipes pueden ser muy abundantes (Zárate et al., 2015; Vásquez, 1997). Pachira brevipes también puede ser muy abundante en los varillales sobre pantanos (Kelly et al., 2014) y algunas especies pueden ser compartidas con los varillales sobre arena blanca, como Platycarpum loretensis (Dávila \& Kinoshita, 2016). Las especies especialistas de varillales de arena blanca incluyen a 23 especies de aves (Álvarez et al., 2010), sin embargo, no hay reportes de especialistas en cuanto a los anfibios, reptiles o mamíferos (Soini \& Álvarez, 2002), ni estudios sobre fauna en varillales hidromórficos.

Los varillales sobre pantano fueron recientemente reportados en un estudio sobre las reservas de carbono debajo del suelo en Loreto (Draper et al., 2014), sin embargo, el estudio incluye de manera parcial la distribución de estos varillales en la provincia de Maynas. En el caso de los varillales sobre arena blanca han sido estudiados desde un punto de macro comunidades (Josse et al., 2007), florístico (Encarnación, 1993; García et al., 2003; Fine et al., 2010; Zárate et al., 2013) y ecológico (Fine et al., 2006; 2013), sin embargo, se desconoce aún su distribución espacial en el departamento de Loreto. La distribución de los varillales se puede obtener usando la teledetección integrada a métodos de SIG e imágenes de satélite de alta resolución (Lu, 2006). Por ejemplo, las imágenes WorldView-2, IKONOS o RapidEye están disponibles con una resolución espacial menor a 5 metros, lo que permite realizar el mapeo de parámetros estructurales y composición de especies vegetales, así como la estructura de la copa de árboles aplicando algoritmos clasificadores (Dube et al., 2014; Palace et al., 2008; Mallinis et al., 2008; Johansen \& Phinn, 2006). Las imágenes RapidEye cuentan con bandas estratégicamente colocadas que permiten elaborar una mejor cartografía de la vegetación (Dube et al., 2014). Así también, estas imágenes cuentan con la banda Borde Rojo (BR) que permite calcular valores exactos del índice de vegetación normalizado (NDVI), con lo cual se puede diferenciar la estructura de los tipos de vegetación (Marx, 2010). Esto se debe a que la banda BR es sensible al contenido de clorofila y un aumento en la concentración de clorofila hace variar la pendiente típica del BR versus la banda del infrarrojo cercano (IRC) (Weichelt et al., 2012).

El uso de las bandas espectrales de imágenes de satélite, tanto de media como de alta resolución, permite caracterizar las propiedades biofísicas y geofísicas de la vegetación, así como la relación entre estas propiedades (Roy et al., 2014; MoraDelgado \& Serrano, 2012; Hansen \& Loveland, 2012;). Uno de los métodos de clasificación supervisada muy utilizado en teledetección es el de Máxima Verosimilitud (MVS), donde primero se definen los valores espectrales de cada categoría de clasificación, asignando después, con los valores espectrales de los pixeles, una clase basada en el valor de mayor probabilidad (Ustuner \& Balik, 2015).

El objetivo del presente trabajo fue elaborar el mapa de los bosques tipo varillal sobre arena blanca y sobre pantano utilizando imágenes de satélite RapidEye en la provincia Maynas. Esta información cartográfica va a permitir una mejor gestión de estas áreas, incluyendo la conservación y uso sostenible de los recursos naturales, en bien de las poblaciones locales que ancestralmente lo habitan.

\section{MATERIAL Y MÉTODOS}

\section{ÁREA DE ESTUDIO}

El área de estudio correspondió a la provincia Maynas $\left(75^{\circ} 47^{\prime} 24.26^{\prime \prime} \mathrm{O}, 0^{\circ} 38^{\prime} 21.97 " \mathrm{~S}\right.$ y $\left.72^{\circ} 6^{\prime} 40.40^{\prime \prime} \mathrm{O}, 4^{\circ} 42^{\prime} 23.41^{\prime \prime S}\right)$, ubicada en el departamento de Loreto, en la zona Nororiental del Perú (Figura 1). La provincia de Maynas limita al 
norte con la República de Colombia, al este con la provincia de Mariscal Ramón Castilla, al sur con la provincia de Requena y al oeste con la provincia de Loreto y la República del Ecuador.

El clima es cálido húmedo tropical, la temperatura media anual es superior a $25{ }^{\circ} \mathrm{C}$. Fisiográficamente presenta terrazas bajas, terrazas medias, terrazas altas y colinas bajas. Los suelos más antiguos ubicados en las colinas y terrazas no inundables son más ácidos, de menor fertilidad, arcillosos o con arenas cuarzosas muy lixiviados (Kauffman et al., 1998). Estos suelos son conocidos como de arena blanca, lugar donde se desarrollan los varillales.

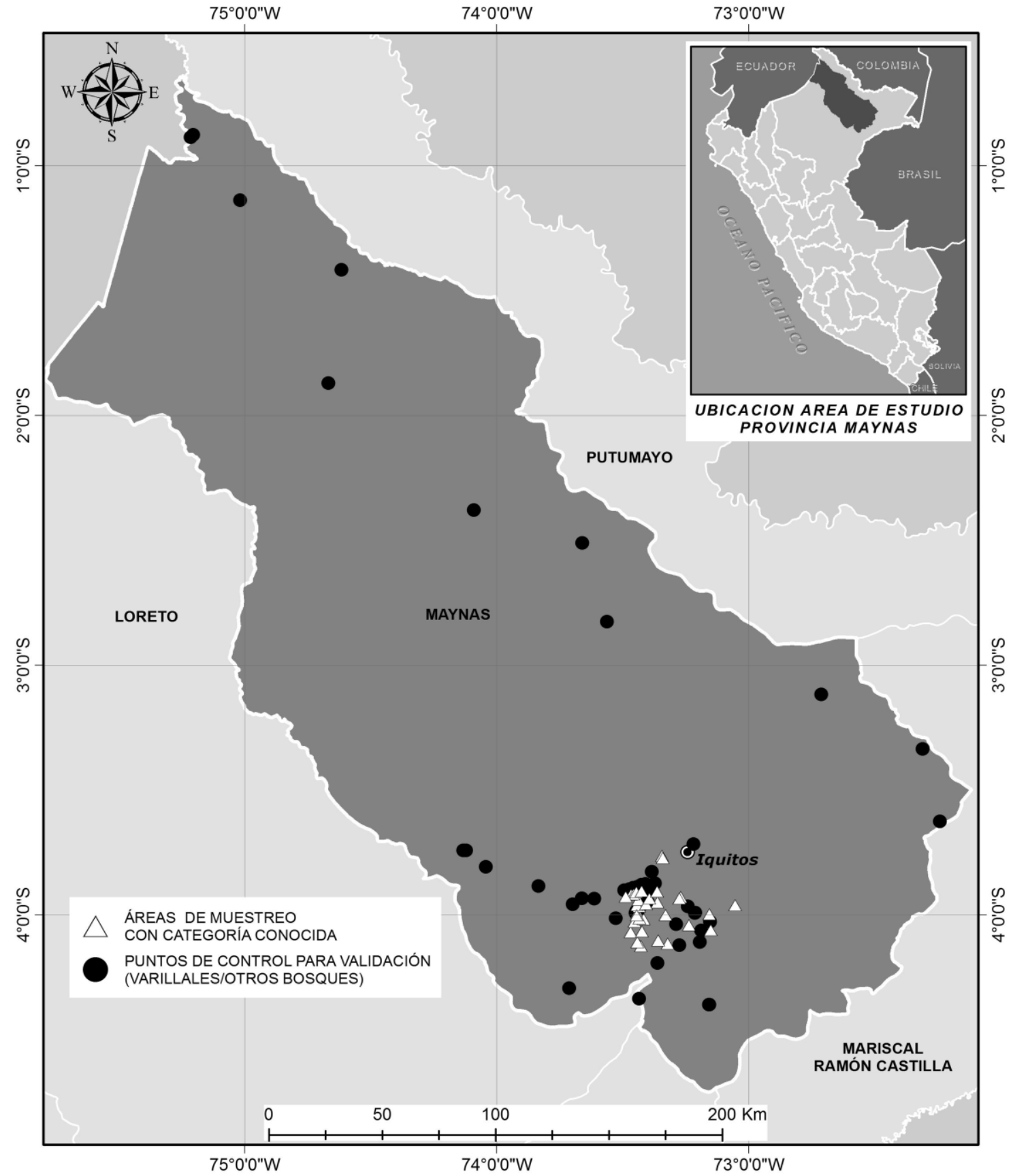

Figura 1. Ubicación de los sitios de muestreo para la elaboración del mapa de varillales de la provincia Maynas, Loreto, Perú. Se registran las 32 áreas con categoría de clasificación conocida y los 57 puntos control usados en la validación de la clasificación de los varillales y otros bosques. 


\section{INFORMACIÓN CARTOGRÁFICA YSATELITAL}

Los datos satelitales utilizados corresponden a 209 escenas de imágenes del satélite RapidEye, nivel Ortho 3A, donadas por el Gobierno de Japón al Gobierno de Perú, para ser utilizadas en procesos de Ordenamiento del Territorio y generación de cartografía hasta una escala de $1 / 25,000$, según consideraciones técnicas de la DGOT-MINAM (2014).

Las imagenes presentan una resolución espacial de 5 metros y fueron tomadas en el 2012, contando con corrección geométrica y radiométrica a nivel de sensor, así también se encontraban referenciadas al sistema WGS 84 y compuestas por 5 bandas: azul (B1: 440-510 nm), verde (B2: 520-590 nm), rojo (B3: 630-685 nm), borde rojo (B4: 690-730 nm), e infrarojo cercano (B5: 760-850 nm) (Blackbridge, 2013). Para el procesamiento fueron seleccionadas aquellas imágenes que tuvieron cobertura de nubes inferior al $10 \%$.

\section{CLASIFICACIÓN DE IMÁGENES}

Las firmas espectrales de los varillales y de otras categorías que caracterizan el paisaje, se obtuvieron usando los niveles digitales (ND) para 32 áreas con información conocida: 8 varillales (Gallardo, 2015), 3 bosques de otro tipo, 2 de arena, 4 de cuerpos de agua, 12 sin vegetación, 1 nube y 1 de sombra (Figura 1). Las 32 áreas están ubicadas en tres imágenes de satélite RapidEye (Tiles: 1837322, 1837223 y 1837222 ) que corresponden a 371,081 píxeles (Tabla 2).

Para definir la consistencia de las áreas de entrenamiento de las categorías aplicamos la interpretación bidimensional comparando las bandas R, BR e IRC (Palacios et al., 2015; Gilabert et al., 1997). Medimos si existía diferencia significativa en los ND expresados en valores de reflectividad para las categorías varillales y otros bosques usando la prueba de Mann-Whitney. Estas pruebas fueron realizadas en el Programa SigmaPlot.
Se generó un archivo de firmas espectrales, que corresponde a una descripción estadística (promedio, matriz de varianza y covarianza) de cada categoría de clasificación (Mathur \& Foody, 2008). Luego, se realizó un análisis de conglomerados con la finalidad de comprobar la máxima homogeneidad de cada categoría y la mayor diferencia entre las categorías propuestas (Bonn \& Rochon, 1992; Chuvieco \& Congalton, 1988), mostrándose en un diagrama de árbol (dendrograma). Los pixeles de cada una de las 206 imágenes fueron clasificados usando las firmas espectrales de las siete categorías, aplicando el algoritmo de Máxima Verosimilitud. El proceso se automatizó en ModelBuilder en el programa ArcGIS versión 10.1.

\section{GENERACIÓN DEL MAPA DEVARILLALES}

Para la generación del mapa de varillales de la provincia de Maynas se aplicó un filtro de tipo "Majority", el cual reemplazó la categoría de píxeles aislados a una categoría de los pixeles más próximos y abundantes, reduciendo así el patrón de pixeles aislados conocido como efecto "sal y pimienta" (Stuckens et al., 2000). Seguidamente, se seleccionó por atributos solo los píxeles de la clase varillales, migrando estos a formato vector y tipo de objeto polígono. Se calculó la superficie en hectáreas de cada polígono y se excluyeron aquellos con áreas menores a 20 hectáreas, teniendo en cuenta el criterio de área mínima cartografiable para la escala 1/100,000 (Priego-Santander et al., 2008; Forbes et al., 1982; Salitchev, 1979).

\section{VALIDACIÓN DEL MAPA}

Se realizó una evaluación de la precisión total de la clasificación Liu et al. (2007), de las categorías varillales y otros tipos de bosque, usando 57 puntos de control que corresponden a 24 puntos de varillales y 33 puntos de otros bosques. Estos puntos provenían de dos fuentes: datos propios tomados en campo (28 puntos) y citados en las publicaciones de Álvarez et al., 2010 y Fine et al., 2010 (29 puntos).

Tabla 1. Análisis de exactitud de las categorías con vegetación usando 57 puntos de control de varillales y otros bosques para la validación del mapa de varillales de la provincia Maynas.

\begin{tabular}{c|cc|c}
\hline \multirow{2}{*}{ Categorías probables } & \multicolumn{2}{|c|}{ Categorías reales } & \multirow{2}{*}{ Total } \\
\cline { 2 - 3 } & Varillales & Otros Bosques & \\
\hline Varillales & 23 & 1 & 24 \\
Otros Bosques & 4 & 29 & 33 \\
Total & 27 & 30 & 57 \\
\hline
\end{tabular}




\section{RESULTADOS}

Los varilalles de la Provincia de Maynas cubren una superficie de 51,024.86 hectáreas, que representan el $0,67 \%$ de la superficie de la provincia (Figura 2). Estos varillales se distribuyen principalmente en la cuenca del río Napo, cuenca baja del río Curaray, cuencas de los ríos Nanay, Chambira y Pintuyacu, margen derecho del río Amazonas (frente a la ciudad de Iquitos) y al este de la confluencia de los ríos Marañón y Ucayali (distrito de Fernando Lores).

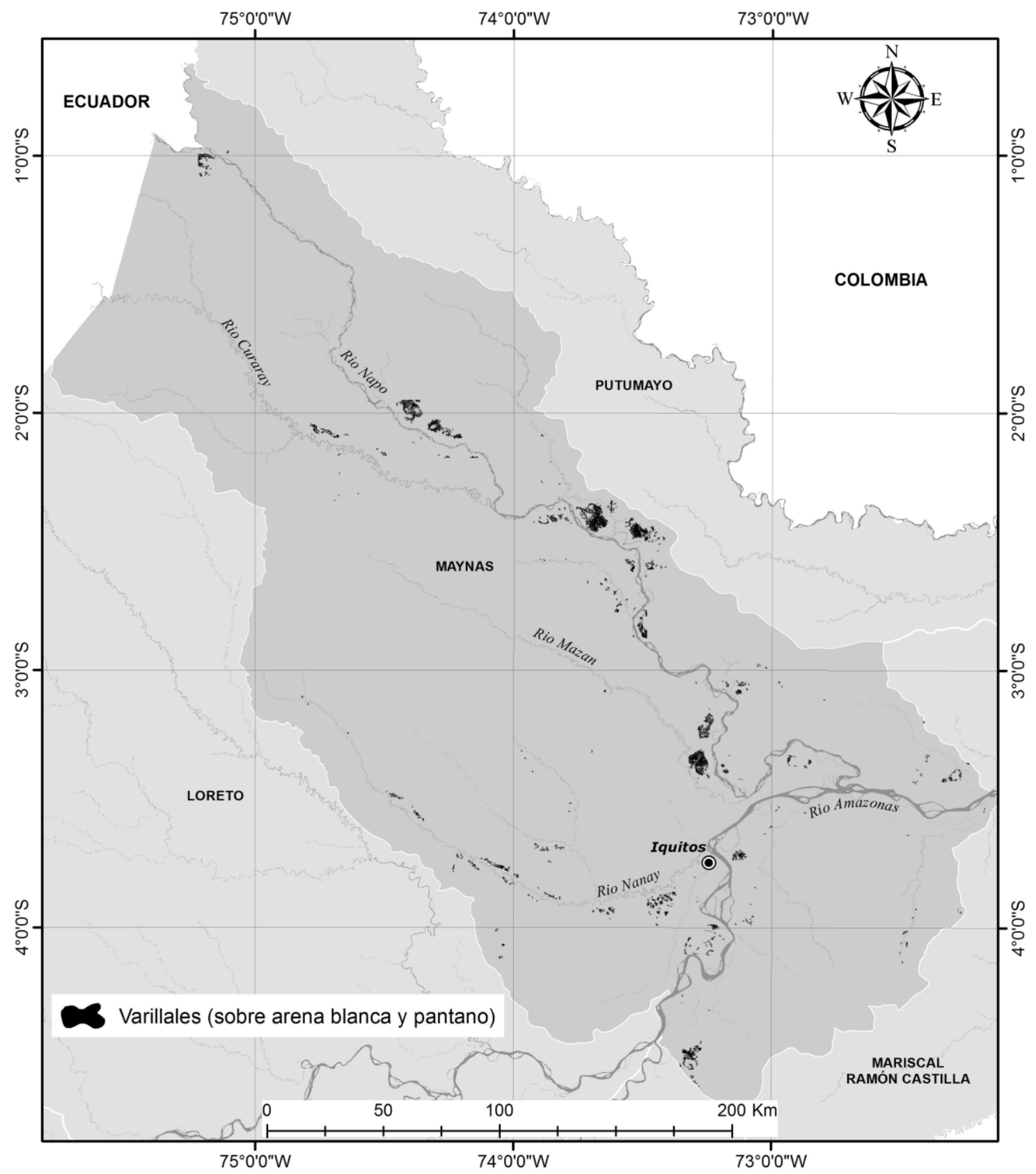

Figura 2. Mapa de la distribución de los bosques tipo varillal sobre arena blanca y sobre pantano en la Provincia Maynas, Loreto, Perú. 

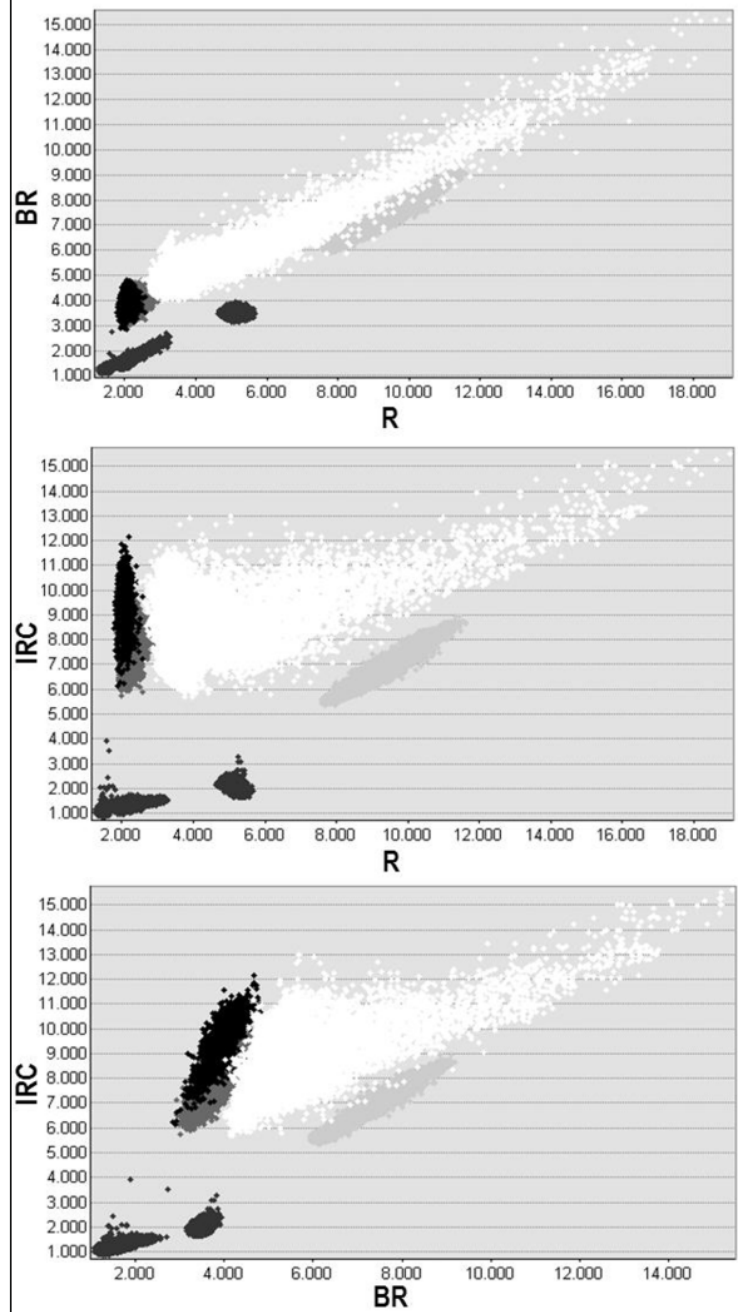

$B R$

\begin{tabular}{cccc}
\hline CÓDIGO & CATEGORÍA & SímBOLO & CANTIDAD DE PIXELES \\
\hline 1 & Varillales & 57063 \\
2 & Otros bosques & & 15822 \\
3 & Arena & 49974 \\
99 & Cuerpo de agua & 211115 \\
100 & Sin vegetación & 32878 \\
\hline
\end{tabular}

Figura 3a
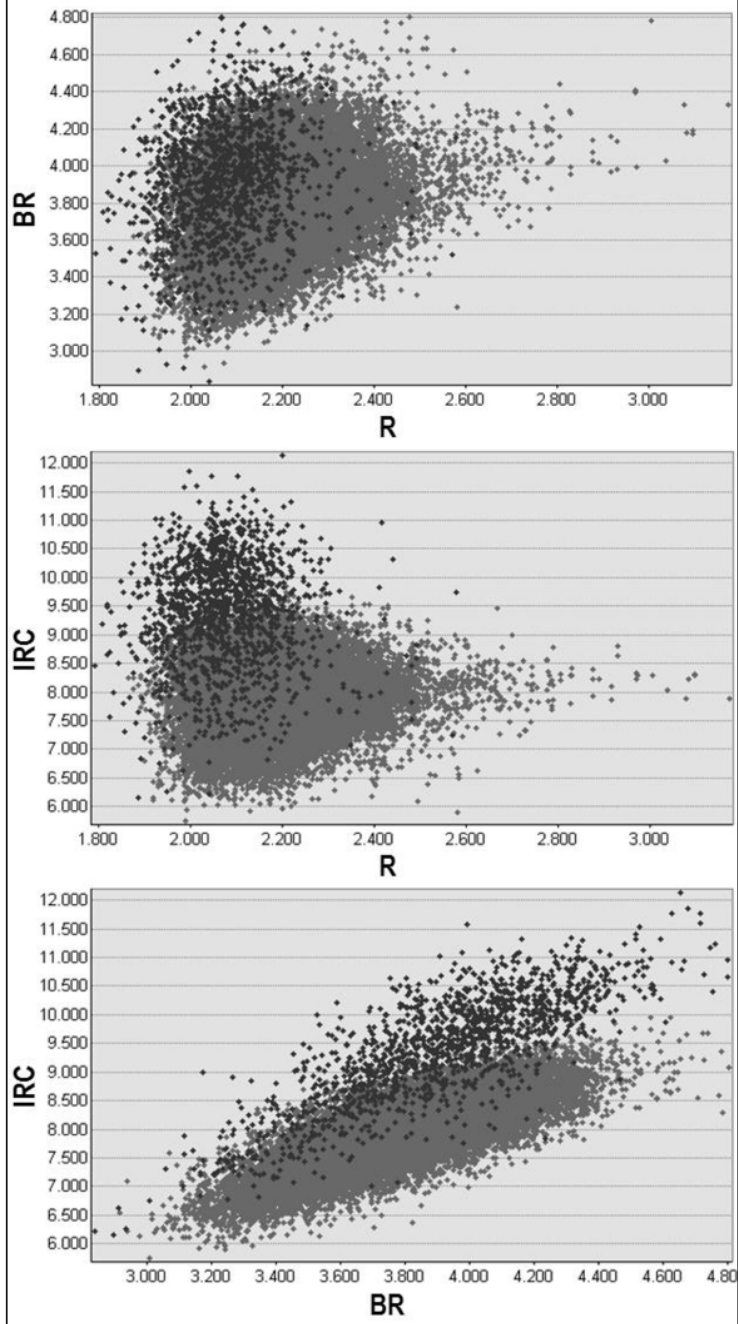

\begin{tabular}{cccc}
\hline CÓdIGO & CATEGORÍA & SÍMBOLO & CANTIDAD DE PIXELES \\
\hline 1 & Varillales & \\
2 & Otros bosques & & 157063 \\
& & \\
\end{tabular}

Figura $3 b$

Figura 3. Interpretación bidimensional de las bandas R, BR, IRC de los niveles digitales de las áreas de entrenamiento. a. Comparación entre todas las categorías propuestas. b. comparación de categorías varillal y otros bosques. 
Existe una distribución diferenciada de las siete categorías en los planos IRC-R e IRC-BR (Figura 3a). En particular, estas combinaciones de bandas muestran una diferencia marcada entre las categorías varillales y otros bosques, mientras los niveles digitales de las muestras de estas categorías en el plano BR-R se sobreponen (Figura 3b). Los valores de reflectividad de los bosques tipo varillal y otros bosques muestran diferencia significativa en todas las bandas (Mann-Whitney, $\mathrm{U}_{\text {AzUL }}=-4.2 \times 10^{10}$, $\mathrm{U}_{\text {VERDE }}=-4.1 \times 10^{10}, \mathrm{H}_{\text {ROJO }}=-4,6 \times 10^{10}, \mathrm{H}_{\mathrm{BR}}=-3.5 \mathrm{x}$ $10^{10}, \mathrm{H}_{\mathrm{IRC}}=-3.1 \times 10^{10}$, todos $\left.\mathrm{p}<0.001\right)$.

Al observar el dendrograma de similitud entre las categorías de clasificación, observamos que las categorías con vegetación, tales como varillales y otros bosques, son más similares entre ellas, diferentes a las categorías sin vegetación, cuerpos de agua, arena, nubes y sombra (Figura 4).

En cuanto a la validación del mapa, tenemos que de los 57 puntos de control, 53 correspondían a las categorías varillales $\mathrm{u}$ otros bosques y 4 no coincidieron, indicando un $91.2 \%$ de precisión (Tabla 1).

\section{DISCUSIÓN}

De acuerdo a nuestros resultados, el uso de imágenes RapidEye permite determinar la distribución espacial de los varillales. Esto se debe a que estas imágenes cuentan con bandas estratégicamente colocadas y la banda Borde Rojo que permite detectar diferencias en la estructura de la vegetación (Dube et al., 2014).

Entonces, se logra diferenciar la estructura particular de los varillales de dosel de menor estatura en comparación a los bosques aledaños que son más altos y en algunos casos frondosos (bosques de tierra firme, aguajales, bosques inundables).

La alta resolución de las imágenes permite detectar también estas formaciones de varillales que ocurren en pequeños parches en el área de estudio. Un resultado similar fue obtenido usando imágenes ALOS-PALSAR que tienen la misma particularidad de poder detectar varillales y diferenciar la estructura del bosque (Draper et al., 2014).

La diferenciación de los varillales con otros tipos de bosque, probablemente también se debe a la presencia de especies que solo ocurren en los varillales con similar físiología (estado de la clorofila) y fenología (Weichelt et al., 2012). Por ejemplo, las especies Pachira brevipes y Dendropanax umbellatus son comunes y abundantes en ambos tipos de varillales (Zárate et al., 2015; Zárate et al., 2013) y no están presentes en los otros tipos de bosques.

Nuestro mapa de distribución de los varillales contiene tanto a los varillales sobre pantano del Río Amazonas, reportados anteriormente por Draper et al. (2014) y a los varillales sobre arena blanca de la carretera Iquitos-Nauta, reportados por Zárate et al. (2013) para la provincia Maynas. Nuestro estudio no tuvo el objetivo de diferenciar estos dos tipos de

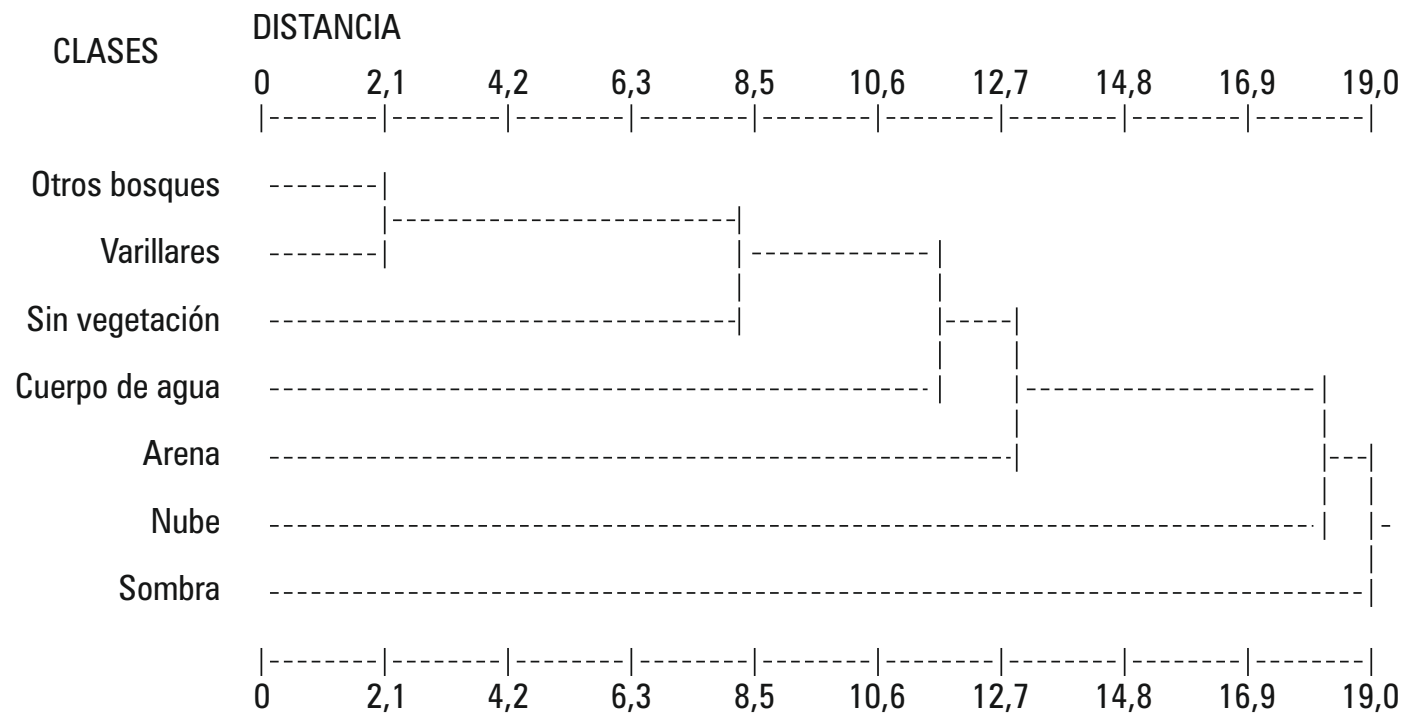

Figura 4. Dendrograma de similitud entre las categorías de clasificación. Se observa que las categorías con vegetación, varillales y otros bosques, son diferentes a las categorías sin vegetación. 
Anexo 1. Ubicación de los 57 puntos de control de las categorías varillales (Var) y otros bosques (OB) usados en la validación del mapa de varillales de la provincia Maynas (Tipo 1). Se incluye la ubicación de los ocho varillales muestreados usando parcelas en campo (Tipo 2).

\begin{tabular}{|c|c|c|c|c|c|c|c|c|c|c|c|}
\hline $\mathrm{N}^{0}$ & Tipo & Cat. & Longitud & Latitud & Fuente & $\mathrm{N}^{0}$ & Tipo & Cat. & Longitud & Latitud & Fuente \\
\hline 1 & 1 & $\mathrm{OB}$ & $73^{\circ} 11^{\prime} 22^{\prime \prime} 0$ & $4^{\circ} 03^{\prime} 41^{\prime \prime} S$ & $\mathrm{Pr}$ & 34 & 2 & Var & $73^{\circ} 27^{\prime} 28^{\prime \prime} 0$ & $3^{\circ} 55^{\prime} 02^{\prime \prime} \mathrm{S}$ & $\mathrm{Pr}$ \\
\hline 2 & 1 & $\mathrm{OB}$ & $73^{\circ} 26^{\prime} 47^{\prime \prime} 0$ & $3^{\circ} 59^{\prime} 04^{\prime \prime} \mathrm{S}$ & $\operatorname{Pr}$ & 35 & 2 & Var & $73^{\circ} 26^{\prime} 51^{\prime \prime} 0$ & $3^{\circ} 54^{\prime} 32^{\prime \prime} S$ & $\operatorname{Pr}$ \\
\hline 3 & 1 & $\mathrm{OB}$ & $73^{\circ} 26^{\prime} 43^{\prime \prime} 0$ & $3^{\circ} 59^{\prime} 35^{\prime \prime} S$ & $\operatorname{Pr}$ & 36 & 2 & Var & $73^{\circ} 25^{\prime} 33^{\prime \prime} 0$ & $3^{\circ} 54^{\prime} 20^{\prime \prime} S$ & $\operatorname{Pr}$ \\
\hline 4 & 1 & OB & $73^{\circ} 26^{\prime} 34^{\prime \prime} 0$ & $3^{\circ} 59^{\prime} 59^{\prime \prime} S$ & $\operatorname{Pr}$ & 37 & 1 & $\mathrm{OB}$ & $73^{\circ} 24^{\prime} 00^{\prime \prime} 0$ & $3^{\circ} 57^{\prime} 02^{\prime \prime} \mathrm{S}$ & $\mathrm{F}$ \\
\hline 5 & 1 & $\mathrm{OB}$ & $73^{\circ} 26^{\prime} 30^{\prime \prime} 0$ & $4^{\circ} 00^{\prime} 04^{\prime \prime} S$ & $\mathrm{Pr}$ & 38 & 1 & $\mathrm{OB}$ & $74^{\circ} 07^{\prime} 58^{\prime \prime} 0$ & $3^{\circ} 44^{\prime} 29^{\prime \prime} S$ & $\mathrm{~F}$ \\
\hline 6 & 1 & OB & $73^{\circ} 41^{\prime} 57^{\prime \prime} 0$ & $3^{\circ} 57^{\prime} 26^{\prime \prime} S$ & $\operatorname{Pr}$ & 39 & 1 & $\mathrm{OB}$ & $72^{\circ} 42^{\prime} 45^{\prime \prime} 0$ & $3^{\circ} 07^{\prime} 00^{\prime \prime} S$ & $\mathrm{~F}$ \\
\hline 7 & 1 & $\mathrm{OB}$ & $73^{\circ} 42^{\prime} 46^{\prime \prime} 0$ & $4^{\circ} 17^{\prime} 37^{\prime \prime} \mathrm{S}$ & $\operatorname{Pr}$ & 40 & 1 & $\mathrm{OB}$ & $74^{\circ} 05^{\prime} 29^{\prime \prime} 0$ & $2^{\circ} 22^{\prime} 43^{\prime \prime} S$ & $\mathrm{~F}$ \\
\hline 8 & 1 & $\mathrm{OB}$ & $73^{\circ} 14^{\prime} 30^{\prime \prime} 0$ & $3^{\circ} 57^{\prime} 54^{\prime \prime S}$ & $\operatorname{Pr}$ & 41 & 1 & $\mathrm{OB}$ & $74^{\circ} 40^{\prime} 04^{\prime \prime} 0$ & $1^{\circ} 52^{\prime} 10^{\prime \prime S}$ & $\mathrm{~F}$ \\
\hline 9 & 1 & $\mathrm{OB}$ & $73^{\circ} 31^{\prime} 41^{\prime \prime} 0$ & $4^{\circ} 00^{\prime} 46^{\prime \prime} S$ & $\operatorname{Pr}$ & 42 & 1 & $\mathrm{OB}$ & $72^{\circ} 14^{\prime} 34^{\prime \prime} 0$ & $3^{\circ} 37^{\prime} 29^{\prime \prime} S$ & $\mathrm{~F}$ \\
\hline 10 & 1 & OB & $73^{\circ} 23^{\prime} 05^{\prime \prime} 0$ & $3^{\circ} 49^{\prime} 39^{\prime \prime} S$ & $\operatorname{Pr}$ & 43 & 1 & $\mathrm{OB}$ & $75^{\circ} 12^{\prime} 53^{\prime \prime} 0$ & $0^{\circ} 53^{\prime} 05^{\prime \prime} S$ & $\mathrm{~F}$ \\
\hline 11 & 1 & $\mathrm{OB}$ & $73^{\circ} 13^{\prime} 14^{\prime \prime} 0$ & $3^{\circ} 42^{\prime} 58^{\prime \prime} S$ & $\operatorname{Pr}$ & 44 & 1 & $\mathrm{OB}$ & $75^{\circ} 12^{\prime} 20^{\prime \prime} 0$ & $0^{\circ} 52^{\prime} 31^{\prime \prime} \mathrm{S}$ & $\mathrm{F}$ \\
\hline 12 & 1 & $\mathrm{OB}$ & $73^{\circ} 26^{\prime} 07^{\prime \prime} 0$ & $4^{\circ} 20^{\prime} 05^{\prime \prime} S$ & $\operatorname{Pr}$ & 45 & 1 & $\mathrm{OB}$ & $73^{\circ} 09^{\prime} 32^{\prime \prime} 0$ & $4^{\circ} 21^{\prime} 33^{\prime \prime} \mathrm{S}$ & $\mathrm{F}$ \\
\hline 13 & 1 & $\mathrm{OB}$ & $73^{\circ} 21^{\prime} 47^{\prime \prime} 0$ & $4^{\circ} 11^{\prime} 33^{\prime \prime} S$ & $\operatorname{Pr}$ & 46 & 1 & $\mathrm{OB}$ & $73^{\circ} 09^{\prime} 26^{\prime \prime} 0$ & $4^{\circ} 21^{\prime} 33^{\prime \prime} \mathrm{S}$ & $\mathrm{F}$ \\
\hline 14 & 1 & $\mathrm{OB}$ & $73^{\circ} 16^{\prime} 34^{\prime \prime} 0$ & $4^{\circ} 07^{\prime} 13^{\prime \prime S}$ & $\operatorname{Pr}$ & 47 & 1 & $\mathrm{OB}$ & $72^{\circ} 18^{\prime} 40^{\prime \prime} 0$ & $3^{\circ} 20^{\prime} 07^{\prime \prime} \mathrm{S}$ & $\mathrm{F}$ \\
\hline 15 & 1 & $\mathrm{OB}$ & $73^{\circ} 11^{\prime} 43^{\prime \prime} 0$ & $4^{\circ} 06^{\prime} 30^{\prime \prime} \mathrm{S}$ & $\operatorname{Pr}$ & 48 & 1 & $\mathrm{OB}$ & $73^{\circ} 39^{\prime} 39^{\prime \prime} 0$ & $2^{\circ} 30^{\prime} 38^{\prime \prime} S$ & $\mathrm{~F}$ \\
\hline 16 & 1 & $\mathrm{OB}$ & $73^{\circ} 09^{\prime} 16^{\prime \prime} 0$ & $4^{\circ} 01^{\prime} 37^{\prime \prime} \mathrm{S}$ & $\operatorname{Pr}$ & 49 & 1 & $\mathrm{OB}$ & $74^{\circ} 36^{\prime} 59^{\prime \prime} 0$ & $1^{\circ} 24^{\prime} 58^{\prime \prime} S$ & $\mathrm{~F}$ \\
\hline 17 & 1 & $\mathrm{OB}$ & $73^{\circ} 17^{\prime} 17^{\prime \prime} 0$ & $4^{\circ} 02^{\prime} 12^{\prime \prime} S$ & $\mathrm{Pr}$ & 50 & 1 & $\mathrm{OB}$ & $73^{\circ} 33^{\prime} 49^{\prime \prime} 0$ & $2^{\circ} 49^{\prime} 33^{\prime \prime} S$ & $\mathrm{~F}$ \\
\hline 18 & 1 & Var & $74^{\circ} 02^{\prime} 36^{\prime \prime} 0$ & $3^{\circ} 48^{\prime} 26^{\prime \prime} S$ & $\operatorname{Pr}$ & 51 & 1 & $\mathrm{OB}$ & $75^{\circ} 01^{\prime} 06^{\prime \prime} 0$ & $1^{\circ} 08^{\prime} 14^{\prime \prime} S$ & $\mathrm{~F}$ \\
\hline 19 & 1 & Var & $73^{\circ} 50^{\prime} 07^{\prime \prime} 0$ & $3^{\circ} 53^{\prime} 01^{\prime \prime} \mathrm{S}$ & $\operatorname{Pr}$ & 52 & 1 & $\mathrm{OB}$ & $73^{\circ} 24^{\prime} 42^{\prime \prime} 0$ & $3^{\circ} 56^{\prime} 52^{\prime \prime} S$ & $\mathrm{~F}$ \\
\hline 20 & 1 & Var & $73^{\circ} 23^{\prime} 19^{\prime \prime} 0$ & $3^{\circ} 53^{\prime} 07^{\prime \prime} \mathrm{S}$ & $\operatorname{Pr}$ & 53 & 1 & Var & $74^{\circ} 07^{\prime} 20^{\prime \prime} 0$ & $3^{\circ} 44^{\prime} 27^{\prime \prime} \mathrm{S}$ & $\mathrm{F}$ \\
\hline 21 & 1 & Var & $73^{\circ} 22^{\prime} 21^{\prime \prime} 0$ & $3^{\circ} 52^{\prime} 22^{\prime \prime} \mathrm{S}$ & $\mathrm{Pr}$ & 54 & 1 & Var & $73^{\circ} 25^{\prime} 35^{\prime \prime} 0$ & $3^{\circ} 52^{\prime} 42^{\prime \prime} S$ & $A$ \\
\hline 22 & 1 & Var & $73^{\circ} 12^{\prime} 47^{\prime \prime} 0$ & $3^{\circ} 59^{\prime} 24^{\prime \prime} S$ & $\operatorname{Pr}$ & 55 & 1 & $\mathrm{OB}$ & $73^{\circ} 24^{\prime} 44^{\prime \prime} 0$ & $3^{\circ} 57^{\prime} 05^{\prime \prime} S$ & $A$ \\
\hline 23 & 1 & Var & $73^{\circ} 12^{\prime} 48^{\prime \prime} 0$ & $3^{\circ} 59^{\prime} 26^{\prime \prime} S$ & $\operatorname{Pr}$ & 56 & 1 & Var & $73^{\circ} 24^{\prime} 39^{\prime \prime} 0$ & $3^{\circ} 52^{\prime} 34^{\prime \prime} \mathrm{S}$ & A \\
\hline 24 & 1 & Var & $73^{\circ} 12^{\prime} 52^{\prime \prime} 0$ & $3^{\circ} 59^{\prime} 32^{\prime \prime} \mathrm{S}$ & $\operatorname{Pr}$ & 57 & 1 & Var & $73^{\circ} 25^{\prime} 32^{\prime \prime} 0$ & $3^{\circ} 54^{\prime} 03^{\prime \prime} S$ & A \\
\hline 25 & 1 & Var & $73^{\circ} 25^{\prime} 27^{\prime \prime} 0$ & $3^{\circ} 57^{\prime} 35^{\prime \prime} \mathrm{S}$ & $\operatorname{Pr}$ & 58 & 1 & Var & $73^{\circ} 26^{\prime} 31^{\prime \prime} 0$ & $3^{\circ} 53^{\prime} 15^{\prime \prime} S$ & $A$ \\
\hline 26 & 1 & Var & $73^{\circ} 26^{\prime} 49^{\prime \prime} 0$ & $3^{\circ} 59^{\prime} 05^{\prime \prime} S$ & $\operatorname{Pr}$ & 59 & 1 & Var & $73^{\circ} 29^{\prime} 41^{\prime \prime} 0$ & $3^{\circ} 54^{\prime} 05^{\prime \prime} S$ & $A$ \\
\hline 27 & 1 & Var & $73^{\circ} 26^{\prime} 41^{\prime \prime} 0$ & $3^{\circ} 59^{\prime} 27^{\prime \prime} \mathrm{S}$ & $\operatorname{Pr}$ & 60 & 1 & Var & $73^{\circ} 39^{\prime} 44^{\prime \prime} 0$ & $3^{\circ} 56^{\prime} 01^{\prime \prime} \mathrm{S}$ & A \\
\hline 28 & 1 & Var & $73^{\circ} 26^{\prime} 48^{\prime \prime} 0$ & $3^{\circ} 59^{\prime} 33^{\prime \prime} S$ & $\operatorname{Pr}$ & 61 & 1 & Var & $73^{\circ} 36^{\prime} 50^{\prime \prime} 0$ & $3^{\circ} 56^{\prime} 04^{\prime \prime} S$ & $A$ \\
\hline 29 & 2 & Var & $73^{\circ} 26^{\prime} 34^{\prime \prime} 0$ & $3^{\circ} 59^{\prime} 25^{\prime \prime} \mathrm{S}$ & $\operatorname{Pr}$ & 62 & 1 & Var & $73^{\circ} 28^{\prime} 32^{\prime \prime} 0$ & $3^{\circ} 53^{\prime} 48^{\prime \prime} S$ & $A$ \\
\hline 30 & 2 & Var & $73^{\circ} 26^{\prime} 11^{\prime \prime} 0$ & $3^{\circ} 57^{\prime} 59^{\prime \prime} \mathrm{S}$ & $\operatorname{Pr}$ & 63 & 1 & Var & $73^{\circ} 27^{\prime} 34^{\prime \prime} 0$ & $3^{\circ} 53^{\prime} 28^{\prime \prime} S$ & $A$ \\
\hline 31 & 2 & Var & $73^{\circ} 26^{\prime} 39^{\prime \prime} 0$ & $3^{\circ} 57^{\prime} 40^{\prime \prime} S$ & $\operatorname{Pr}$ & 64 & 1 & Var & $73^{\circ} 26^{\prime} 56^{\prime \prime} 0$ & $3^{\circ} 59^{\prime} 30^{\prime \prime} S$ & $A$ \\
\hline 32 & 2 & Var & $73^{\circ} 26^{\prime} 17^{\prime \prime} 0$ & $3^{\circ} 56^{\prime} 23^{\prime \prime} S$ & $\operatorname{Pr}$ & 65 & 1 & Var & $73^{\circ} 24^{\prime} 04^{\prime \prime} 0$ & $3^{\circ} 54^{\prime} 42^{\prime \prime} S$ & $A$ \\
\hline 33 & 2 & Var & $73^{\circ} 28^{\prime} 06^{\prime \prime} 0$ & $3^{\circ} 55^{\prime} 07^{\prime \prime} \mathrm{S}$ & $\operatorname{Pr}$ & & & & & & \\
\hline
\end{tabular}

Fuente: Datos propios (Pr), Fine et al., 2010 (F) y Álvarez et al., 2010 (A). 
varillales, sin embargo, la diferenciación de estos dos tipos de varillales es importante porque los varillales hidromórficos a diferencia de los varillales sobre arena blanca, acumulan gran cantidad de carbono debajo del suelo en forma de turba (Draper et al., 2014). La identificación de estos bosques podría ser importante para definir áreas promisorias para desarrollar proyectos de conservación del carbono para mitigar el cambio climático.

El varillal hidromórfico o varillal sobre pantano fue recientemente reportado y nuestros resultados indican que podría existir una mayor distribución en el área de estudio. Este tipo de bosque ha sido confundido por Josse et al. (2007) y Encarnación et al. (2015) con los bosques de palmeras sobre pantano (localmente conocidos como Aguajales) debido a su alta similitud mediante interpretación visual de imágenes satelitales de resolución espacial media como Landsat. Los varillales sobre arena blanca fueron reportados en varios estudios en los sectores de la Reserva Nacional Allpahuayo Mishana y las cuencas de los ríos Nanay, Pintuyacu y Chambira (Adeney et al., 2016; Álvarez et al., 2010; Fine et al., 2010; Gómez, 2006; Josse et al., 2007; Encarnación, 1993). Este estudio muestra la importancia de utilizar imágenes de satélite de alta resolución como las imágenes RapidEye, así como escalas de mayor detalle respecto a los trabajos anteriormente mencionados. Esto conlleva a la identificación de varillales en sectores anteriormente no reportados para Loreto.

En el análisis de precisión notamos que cuatro varillales fueron clasificados como otros tipos de bosque. Esto se puede deber a que la firma espectral de los varillales es más diversa de lo que hemos logrado capturar con las áreas de entrenamiento, basada en datos de campo de esta categoría, implicando esto que probablemente no incluimos la variación en la estructura de los varillales que en algunos casos puede incluir dosel de mayor altura similar a los bosques de altura. Existen unos pocos varillales sobre arena blanca de la cuenca del río Nanay que no se muestran en nuestro mapa, debido a falta de datos de niveles digitales de las imágenes de satélite disponibles. Este es el primer mapa de la distribución de los varillales de Maynas y por lo tanto, recomendamos realizar futuros trabajos de evaluación en campo para determinar la diferencia espectral de los dos tipos de varillales sobre arena blanca y sobre pantano. Muchas áreas de varillales de la provincia de Maynas no se encuentran conservadas dentro de ningún área natural protegida, lo cual debe ser un tema de prioridad para el Estado y la región Loreto. Finalmente, confirmamos el uso de imágenes RapidEye para clasificar y diferenciar los varillales de otros tipos de bosque utilizando las bandas BR e IRC.

\section{AGRADECIMIENTOS}

Los autores agradecemos al Gobierno de Japón, quien en el marco de la Cooperación Financiera: "Donación Hatoyama", nos donó las imágenes satelitales de la constelación RapidEye del territorio peruano. A la Dirección General de Ordenamiento Territorial del Ministerio del Ambiente (DGOTMINAM) por compartir con el Gobierno Regional de Loreto (GORE LORETO) y el Instituto de Investigaciones de la Amazonia Peruana (IIAP), las escenas de imágenes de satélite correspondientes al departamento de Loreto. A Aldo A. Alva por su colaboración en el análisis estadístico. A los revisores del manuscrito de la presente publicación: Paul Fine, Pontus Olofsson y revisor anónimo por sus notorios aportes.

\section{BIBLIOGRAFÍA CITADA}

Adeney, J. M.; Christensen, N. L.; Vicentini, A.; Cohn-Haft, M. 2016. White-sand Ecosystems in Amazonia. Biotropica, 48(1):7-23.

Álvarez, J.; Díaz, J.; Shany, N. 2010. Avifauna de la Reserva Nacional Allpahuayo Mishana, Loreto, Perú. Cotinga, 34:132-152.

Blackbridge. 2013. Satellite imagery, product $\mathrm{spe}$ cific a tions. h t t p:// w w w . blackbridge.com/rapideye/upload/RE_Product Specifications_ENG.pdf ultimo acceso: Junio 2016).

Bonn, F.; Rochon, G. 1992. Précis de télédétection, Volume 1, Principes et méthodes. Presses de l'Université du Québec. Québec. 486pp.

Chuvieco, E.; Congalton, R. 1988, Using cluster analysis to improve the selection of training statistics in classifying remotely sensed data. Photogrammetric Engineering and Remote Sensing, 54(9): 1275-1281.

Dávila, N.; Kinoshita, L. S. 2016. A new species of Platycarpum (Rubiaceae, Henriquezieae) from Peruvian Amazon. Phytotaxa, 260(3), 276-282.

Dirección General de Ordenamiento Territorial DGOT-MINAM. 2013. GUÍA TÉCNICA PARA EL USO DE LAS IMÁGENES RAPIDEYE. Viceministerio de Desarrollo Estratégico de los Recursos Naturales. Ministerio del Ambiente Perú. Lima. 10pp.

Draper, F.; Roucoux, K.; Lawson, I.; Mitchard, E.; Honorio, E.; Lähteenoja, O.; Torres, L.; Valderrama, E.; Zárate, R.; Baker, T. 2014. The distribution and amount of carbon in the largest peatland complex in Amazonia. Environmental Research Letters, 9:1-12. 
Dube, T.; Mutanga, O.; Elhadi, A.; Ismail, R. 2014 Intra-and-inter species biomass prediction in a plantation forest: testing the utility of high spatial resolution spaceborne multispectral rapideye sensor and advanced machine learning algorithms. Sensors, 14(8), 15348-15370

Encarnación, F.; Zárate, R.; Mori, T. 2015. Temático de Vegetación. Zonificación Ecológica y Económica-ZEE de la provincia Alto Amazonas, departamento Loreto. Iquitos Perú. Instituto de Investigaciones de la Amazonía Peruana. Iquitos, Perú. 56pp.

Encarnación, F. 1993. El bosque y las formaciones vegetales en la llanura amazónica del Perú. Alma Mater, 6: 93-114.

Fine, P.; Mesones, I.; García, R.; Miller, Z.; Daly, D.; Coley, P. 2006. Especialización Edáfica en Plantas de la Amazonía Peruana. Folia Amazónica, 15 (1-2): 39-99.

Fine, P.; García, R.; Pitman, N.; Mesones, I.; Kembel, S. 2010. A Floristic Study of the WhiteSand Forests of Peru. Annals of the Missouri Botanical Garden, 97(3):283-305.

Fine, P.; Metz, M.; Lokvam, J.; Mesones, I.; Ayarza, M.; Lamarre, G.; Vásquez, M.; Baraloto, C. 2013. Insect herbivores, chemical innovation, and the evolution of habitat specialization in Amazonian trees. Ecology, 94(8): 1764-1775.

Fine, P.; Miller, S.; Mesones, I.; Irazuzta, S.; Appel, H., Stevens, H.; Saaksjarvi, I.; Schultz, J.; Colley, P. 2006. The growth-defense trade-off and habitat specialization by plants in amazonian forests. Ecology, 87(7): 150-162.

Forbes, T.; Rossiter, D.; Wambeke, A. 1982. Guidelines for evaluating the adequacy of soil resource inventories. Other. Soil Management Support Services, Ithaca, New York. 51pp.

Gallardo, G. 2015. Estructura y diversidad florística de un bosque sobre arena blanca (varillal) en la Reserva Nacional Allpahuayo Mishana, IquitosPerú. Tesis de grado. Universidad Nacional de la Amazonía Peruana. Iquitos. 115pp.

García, R.; Ahuite, M.; Olórtegui, M. 2003. Clasificación de bosques sobre arena blanca de la Zona Reservada Allpahuayo-Mishana. Folia Amazónica, 14 (1): 18-33.

Gilabert, M. A.; González-Piqueras, J.; GarcíaHaro, J. 1997. Acerca de los índices de vegetación. Revista de teledetección, 8(10): 110.

Gómez, D. 2006. Distribución de «varillales» y «chamizales» en la zona reservada Allpahuayo Mishana (ZRAM) utilizando imágen digital del satélite Landsat tm. Investigaciones Sociales, 17:381-393pp.
Hansen, M. C.; Loveland, T. R. 2012. A review of large area monitoring of land cover change using Landsat data. Remote sensing of Environment, 122, 66-74.

Johansen, K.; Phinn, S. 2006. Mapping structural parameters and species composition of riparian vegetation using IKONOS and Landsat ETM+ data in Australian tropical savannahs. Photogrammetric Engineering \& Remote Sensing, 72(1), 71-80.

Josse, C.; Navarro, G.; Encarnación, F.; Tovar A.; Comer, P.; Ferreira, W.; Rodríguez, F.; Saito, J.; Sanjurjo, J.; Dyson, J.; Rubin de Celis E.; Zárate, R.; Chang, J.; Ahuite, M.; Vargas, C.; Paredes, F.; Castro, W.; Maco J.; Reátegui, F. 2007. Sistemas Ecológicos de la Cuenca Amazónica de Perú y Bolivia. Clasificación y mapeo. NatureServe. Virginia. 94pp.

Kauffman, S.; Paredes, G.; Marquina, R. 1998. Suelos de la Zona de Iquitos. In: Kalliola, R.; Flores, S., (Eds). Geoecología y Desarrollo Amazónico. Estudio Integrado en la zona de Iquitos. p. 139-229.

Kelly, T.J.; Baird, A.J.; Roucoux, K.H.; Baker, T.R.; Honorio Coronado, E.N.; Ríos, M.; Lawson, I.T. 2014. The high hydraulic conductivity of three wooded tropical peat swamps in northeast Peru: measurements and implications for hydrological function. Hydrological Processes, 28: 33733387.

Lu, D. 2006. The potential and challenge of remote sensing-based biomass estimation. International Journal of Remote Sensing, 27(7): 1297-1328.

Mallinis, G.; Koutsias, N.; Tsakiri-Strati, M.; Karteris, M. 2008. Object-based classification using Quickbird imagery for delineating forest vegetation polygons in a Mediterranean test site. ISPRS Journal of Photogrammetry and Remote Sensing, 63(2), 237-250.

Mathur, A.; Foody G.M. 2008. Crop classification by support vector machine with intelligently selected training data for an operational application. International Journal of Remote Sensing, 29(8):2227-2240.

Mora-Delgado, J.; Serrano, J. R. 2012. Interpretación y modelamiento de cobertura arbórea en pasturas con el uso de SIG. Agroforesteria Neotropical, 2: 59-67.

Palace, M.; Keller, M.; Asner, G. P.; Hagen, S.; Braswell, B. 2008. Amazon forest structure from IKONOS satellite data and the automated characterization of forest canopy properties. Biotropica, 40(2), 141-150. 
Palacios, J. J.; Zárate, R.; Torres, G. M.; Macedo, N. L.; Alva, A. A.; Gonzales, P. Y. 2015. Evaluación de la Reflectividad de Herbazales pantanosos y áreas deforestadas utilizando imágenes Landsat 8 en Loreto, Perú. Folia Amazónica, 24 (1): 1-8.

Priego-Santander, A.; Bocco, G.; Mendoza, M.; Garrido, A. 2008. Propuesta para la generación semi automatizada de unidades de paisajes, fundamentos y métodos. Instituto Nacional de Ecología. México. 98pp.

Roy, D.P.; Wulder, M.A.; Loveland, T. R.; Woodcock, C. E.; Allen, R. G.; Anderson, M. C.; Helder, D.; Irons, J. R.; Johnson, D. M.; Kennedy, R.; Scambos, T. A.; Schaaf, C. B.; Schott, J. R.; Sheng, Y.; Vermote, E. F.; Belward, A. S.; Bindschadler, R.; Cohen, W. B.; Gao, F.; Hipple, J. D.; Hostert, P.; Huntington, J.; Justice, C. O.; Kilic, A.; Kovalskyy, V.; Lee, Z. P.; Lymburner, L.; Masek, J. G.; McCorkel, J.; Shuai, Y.; Trezza, R.; Vogelmann, J.; Wynne, R. H.; Zhu, Z. 2014. Landsat-8: Science and product vision for terrestrial global change research. Remote Sensing of Environment, 145: 154-172.

Soini, P.; Álvarez, J. 2002. Diagnóstico actualizado de la fauna silvestre de la Reserva Nacional Allpahuayo Mishana y lineamientos para su conservación y manejo. Instituto de Investigaciones de la Amazonía Peruana. Iquitos. Perú. 34pp.

Salitchev, K. A. 1979. Cartografía: La Habana: Cuba. Ministerio de Educación. Editorial Pueblo y Educación. La Habana. 182pp.

Stuckens, J.; Coppin, P.; Bauer, M. 2000. Integrating contextual information with per-pixel classification for improved land cover classification. Remote Sensing of Environment, 71(3): 282-296.

Ustuner, M.; Balik F. S. 2015. Testing the Sensitivity of Vegetation Indices for Crop Type
Classification Using Rapideye Imagery. Libro de resúmenes de de la FIG Working Week 2015. Sofia. Bulgaria. 9pp.

Vásquez, R. 1997. Flórula de las reservas biológicas de Iquitos, Perú. Monographs in Systematic Botany from the Missouri Botanical Garden, 63. EEUU. 1046pp.

Vicentini, A. 2004. A vegetação ao longo de um gradiente edáfico no Parque Nacional do Jaú. In: Borges, S.H.; Iwanaga, S.; Durigan, C.C.; Pinheiro, M. R. (Eds). Janelas para a biodiversidade no Parque Nacional do Jaú: uma estratégia para o estudo da biodiversidade na Amazônia.p. 105-131.

Weichelt, H.; Rosso, P.; Marx, A.; Reigber, S.; Douglass, K.; Heynen, M. 2012. The RapidEye Red Edge Band.

(http://www.blackbridge.com/rapideye/upload/Red _Edge_White_Paper.pdf). Acceso: 19/10/2015.

Zárate, R.; Amasifuen, C.; Flores, M. 2006. Floración y Fructificación de plantas leñosas en bosques de arena blanca y de suelo arcilloso en la Amazonía Peruana. Revista Peruana de Biología, 13: 095-102.

Zárate, R.; Mori, T.; Maco, J. 2013. Estructura y Composición Florística de las Comunidades Vegetales del ámbito de la Carretera IquitosNauta, Loreto, Perú. Folia Amazónica, 22(1-2): 77-89 p.

Zárate, R.; Mori, T.; Ramírez, F.; Dávila, H.; Gallardo, G.; Cohello, G. 2015. Lista actualizada y clave para la identificación de 219 especies arbóreas de los bosques sobre arena blanca de la Reserva Nacional Allpahuayo Mishana, Loreto, Perú. Acta Amazonica, 45(2):133- 156 .

Recibido: 1 de Febrero del 2016

Aceptado para publicación: 29 de Marzo del 2016 
